\title{
Isolated Gust Generation for the Investigation of Airfoil-Gust Interaction
}

\author{
Esteban A. L. Hufstedler* and Beverley J. McKeon ${ }^{\dagger}$ \\ California Institute of Technology, Pasadena, CA, 91125, USA
}

\begin{abstract}
As part of an effort to examine the impact of vortical gusts on airfoils, a simple gust generator has been built and investigated. This consists of a heaving flat plate capable of following a specified transverse trajectory across a water tunnel. The relationship between the trajectory and the properties of the gusts that are shed downstream is characterized for non-periodic heaving motion described by Eldredge's ${ }^{1}$ smooth motion equation. PIV experiments show that the circulation of the vortical gust is proportional to the heaving speed of the plate. Tests with a downstream NACA 0018 airfoil demonstrate repeatable forces in response to the produced gusts.
\end{abstract}

\section{Nomenclature}

$\begin{array}{ll}\alpha_{e f f} & \text { Effective angle of attack of moving plate, arctan }(S) \\ \Delta x & \text { Streamwise distance between plate and airfoil } \\ \Gamma_{v} & \text { Circulation of the shed vortex } \\ \nu & \text { Kinematic viscosity } \\ b & \text { Wetted span of wing, plate } \\ c & \text { Plate chord length } \\ c_{a} & \text { Airfoil chord length } \\ d \cdot c & \text { Plate thickness } \\ H & \text { Heaving distance ratio, } h / c \\ h & \text { Plate heave distance } \\ h_{0} & \text { Initial plate offset from wall } \\ R e & \text { Reynolds number, } U c / \nu \\ S & \text { Heaving speed ratio, } V_{h} / U \\ T & \text { Heaving time ratio, } t_{h e a v e} / t_{c} \\ t & \text { Time } \\ t_{a c c e l} & \text { Time for plate to accelerate to the next speed } \\ t_{c} & \text { Convective time, } c / U \\ t_{h e a v e} & \text { Heaving time, } h / V_{h} \\ t_{\text {peak }} & \text { Point in time where peak motion is reached } \\ t_{s h e d} & \text { von Karman vortex shedding timescale } \\ U & \text { Freestream speed } \\ U_{p} & \text { Total plate speed, } \sqrt{U^{2}+V_{h}^{2}} \\ V_{h} & \text { Plate heave speed } \\ & \end{array}$

\section{Introduction}

Incomplete understanding of the sensitivity of flow around an airfoil to incoming gusts constitutes a limiting factor in the characterization of aerodynamic performance. Improved understanding of this phenomenon

*PhD Candidate, Graduate Aerospace Laboratories of the California Institute of Technology, ehufsted@caltech.edu, AIAA Student Member.

${ }^{\dagger}$ Professor of Aeronautics, Graduate Aerospace Laboratories of the California Institute of Technology, mckeon@caltech.edu, AIAA Associate Fellow. 
could be useful in several contexts. For example, control applications could help reduce the magnitude of lift oscillations on an aircraft or turbine blade, or systems could be designed to harvest energy from these gusts. Since there are many types of gusts, there is no standard method for creating them. This paper investigates the vortical gusts created by a heaving plate, the parameters of which determine the initial position and circulation of the gust.

Studies of the response of airfoils to gusts began with unsteady thin airfoil theory, developed by Wagner, Theodorsen, and others. ${ }^{2-6}$ These analytically investigated sinusoidal and impulsive changes in transverse velocity, as well as impulsive changes in the airfoil's angle of attack. For engineering purposes, the Federal Aviation Administration ${ }^{7}$ defined a reference gust as a change in the freestream velocity scaled with one period of a one-minus-cosine function. This approximation of a gust has been used by Etele ${ }^{8}$ and Moulin, ${ }^{9}$ among others, in a number of computational models of gust response.

Experimentally, many methods of generating gusts have been used. Kerstens et al. ${ }^{10}$ generated an unsteady freestream velocity via actuation of shutters on a wind tunnel. Harmonically pitching the vanes of a tunnel, as performed by Brion et al., ${ }^{11}$ can generate a series of discrete vortices which act as transverse perturbations when far enough from the test article. Using a large number of such vanes, Buell ${ }^{12}$ created streamwise and transverse gusts. Discrete gusts are less frequently created experimentally. One method, used by Perrotta and Jones, ${ }^{13}$ used a set of jets mounted on the bottom of a tow tank to provide a limited region of transverse velocity.

The gust generation method investigated in the current paper is aperiodic, but it can be related to a single period of a harmonically plunging plate. Flow around such a plate is characterized by three dimensionless parameters: ${ }^{14}$ the reduced heaving frequency, advance ratio, and Reynolds number. Analogous parameters for the aperiodic motion will be introduced in section III. The development and detachment of leading edge vortices (LEVs) has been related to these parameters. ${ }^{15}$ For the aperiodic motion, the LEV may be a factor when the plate moves quickly for a relatively short time.

The parameterized equation of motion of the plate is developed in section II. The relative scales of the system are investigated in section III, leading to an estimate of the strength of released vortices. Section IV details the tools used to perform the experiments described in section V. Results of the experiments and comparison to theory are shown in section VI. Conclusions and potential future work are discussed in section VII.

\section{Gust Generation Method}

The proposed method of gust generation involves heaving a thin flat plate in a freestream to create vortical structures that then advect downstream. Equation 1 describes the plate's position over time, using a version of the Eldredge equation ${ }^{1}$ which is plotted in Figure 1 . The motion is parameterized by the starting distance from the wall, $h_{0}$, the heaving distance, $h$, the time to peak displacement, $t_{\text {heave }}$, and the acceleration time, $t_{\text {accel }}$. Here, the variable $a$ was constructed to ensure that the plate completes $95 \%$ of its velocity change over $t_{\text {accel }}$. As shown in Figure 1, small values of $t_{\text {accel }}$ relative to $t_{\text {heave }}$ yield a motion where the plate quickly accelerates from rest to a near-constant speed, $V_{h}$, then retracts with that same speed. Ideally, the rapid change in direction releases a vortex from the plate, with its strength determined by the parameters of motion.

$$
\begin{aligned}
t_{1} & =t_{\text {peak }}-t_{\text {heave }} \\
t_{2} & =t_{\text {peak }} \\
t_{3} & =t_{\text {peak }}+t_{\text {heave }} \\
a & =k / t_{\text {accel }}=2 \operatorname{arctanh}(19 / 20) / t_{\text {accel }} \approx 3.66 / t_{\text {accel }} \\
y_{p}(t) & =h_{0}+\frac{h}{2 a t_{\text {heave }}} \log \left(\frac{\cosh \left(a\left(t-t_{1}\right)\right) \cosh \left(a\left(t-t_{3}\right)\right)}{\cosh \left(a\left(t-t_{2}\right)\right)^{2}}\right) \\
& =h_{0}+\frac{h t_{\text {accel }}}{2 k t_{\text {heave }}} \log \left(1+\sinh \left(\frac{k t_{\text {heave }}}{t_{\text {accel }}}\right)^{2} \cosh \left(\frac{k\left(t-t_{\text {peak }}\right)}{t_{\text {accel }}}\right)^{-2}\right)
\end{aligned}
$$


Plate Position
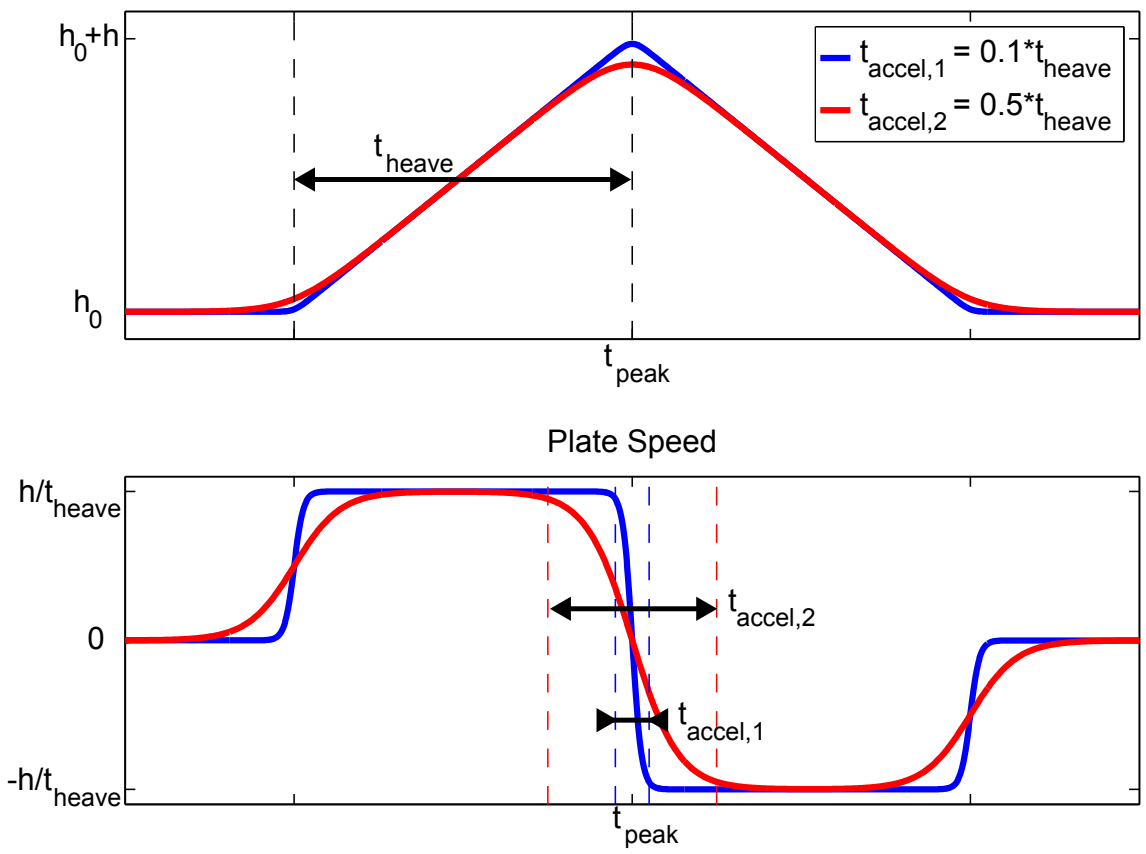

Figure 1: Plate position and speed using the Eldredge function.

\section{Theory}

Examination of the relative scales in this system can help elucidate its behavior. Using the length of the plate, $c$, the ratio of the viscous timescale, $c^{2} / \nu$, to the convection time, $t_{c}$, yields the Reynolds number: $R e$. For the rest of the analysis, it is assumed that $R e \gg 1$. The ratio of the heaving speed to the freestream, $S$, determines the effective angle of attack of the plate in motion, $\tan \left(\left|\alpha_{e f f}\right|\right)=S$, and the plate's total speed, $U_{p}$. The direction of the plate's motion determines the sign of $\alpha_{e f f}$ : negative for upward $(+y)$ plate motion, and positive for downward motion. The motion of the plate provides two more kinematic timescales: the heaving time $t_{\text {heave }}$ and the acceleration time $t_{\text {accel }}$. The timescale associated with shedding von Karman vortices from the moving plate, $t_{\text {shed }}$, can be estimated from the Strouhal number, $S t$, and dimensions of the plate as in Equation 2. The Strouhal number is relatively constant when computed using the projected thickness of the plate, ${ }^{16} w_{p}$, which is a function of its chord-length and thickness ratio, $d$. Together, these yield a number of dimensionless parameters under the experimenter's control: $R e, S, T, t_{\text {accel }} / t_{\text {heave }}$, and $d$.

$$
\begin{aligned}
w_{p} & =c\left(d+\tan \left|\alpha_{e f f}\right|\right) \\
t_{\text {shed }} & =\frac{w_{p}}{S t U_{p}}=\frac{t_{c}}{S t}\left(\frac{S+d \sqrt{1+S^{2}}}{1+S^{2}}\right)
\end{aligned}
$$

The period of the plate moving with constant velocity can be estimated as $t_{\text {heave }}-t_{\text {accel }}$. If this is significantly larger than $t_{\text {shed }}$ the plate may reach a quasi-steady state with regular von Karman vortex shedding. In contrast, if the constant-velocity period is small, then a starting vortex may be attached to the plate when it changes directions. The state of the flow once it has reached that quasi-steady state is determined by $\alpha_{e f f}(S)$. For this geometry, values of $\left|\alpha_{e f f}\right| \lesssim 10^{\circ}$ have attached flow over the plate, whereas higher values may have a large separated region. When the plate changes direction, the behavior of the shed vorticity depends on $t_{\text {accel }} / t_{\text {shed }}$. If this value is large, the shed vorticity may be spread over multiple vortices. Since a single coherent vortex is desired, $t_{a c c e l}$ should be as small as possible. This will also increase the constant-velocity period, helping to avoid starting effects.

Using the terminology of a periodically heaving plate, the analogous reduced frequency and advance ratios can be expressed as $(2 T)^{-1}$ and $S / 2$, respectively. Experiments by Akkala et al. ${ }^{17}$ with such periodic 
motion suggest that values of $T>1$ may result in multiple vortices shedding from the plate in motion, rather than a single starting vortex.

\section{A. Vortical Gust Strength}

The rich body of literature concerning unsteady aerodynamics provides multiple ways to analyze the behavior of this system. The simplest model, however, uses steady thin airfoil theory. ${ }^{18}$ Equation 3 uses the KuttaJoukowski theorem ${ }^{19,20}$ and the thin airfoil theory lift coefficient to estimate the bound circulation of the plate in motion. Using Kelvin's circulation theorem, ${ }^{21}$ the strength of the shed vortex can be approximated as the difference in the plate's circulation before and after its change of direction. Equation 4 estimates this strength as $\Gamma_{v, \text { steady }}$ for a plate moving initially upwards (positive $y$ direction).

$$
\begin{gathered}
L=\rho U_{p} \Gamma_{\text {plate }} b=\frac{1}{2} \rho U_{p}^{2} b c C_{L} \\
C_{L}=2 \pi \alpha_{\text {eff }} \\
\Gamma_{\text {plate }}=\pi c U_{p} \alpha_{\text {eff }}=\pi c U \alpha_{e f f} \sqrt{1+S^{2}} \\
\Gamma_{v, \text { steady }}=\Gamma_{\text {plate }, \text { down }}-\Gamma_{\text {plate }, \text { up }} \\
=2 \pi c U \arctan (S) \sqrt{1+S^{2}}
\end{gathered}
$$

This overestimates the strength of the vortex, as it ignores unsteady and viscous effects. A first estimation of the unsteady effects uses the Wagner Function, an approximation of which is Equation 5. The Wagner function describes how the circulation on a wing approaches its steady state after a step change in angle of attack. Instantaneously after such a step, the circulation changes by half of the difference in the steady lift. The change in direction of motion of the plate can be approximated as a sharp change in angle of attack. Thus, an improved estimate for the vortex strength, $\Gamma_{v}$, is half of the steady-state value, as in Equation 6 . For $S \lesssim 1$, this can be linearized as shown.

$$
\begin{aligned}
& \phi(t) \approx 1-0.165 e^{-0.0455 t / t_{c}}-0.335 e^{-0.3 t / t_{c}} \\
& \phi(0)=0.5 \\
& \Gamma_{v}=\Gamma_{v, \text { steady }} / 2 \\
& =\pi c U \arctan (S) \sqrt{1+S^{2}} \\
& \approx \pi c U S \quad \text { for } \quad S \lesssim 1
\end{aligned}
$$

\section{Experimental Apparatus}

Experiments were performed in the NOAH free-surface water tunnel at Caltech, which has a $46 \mathrm{~cm} \mathrm{x}$ $46 \mathrm{~cm} \mathrm{x} 152 \mathrm{~cm}$ test section. The tunnel can provide speeds of 5 to $30 \mathrm{~cm} / \mathrm{s}$, with a freestream turbulence intensity of under $6 \%$ over that range. A diagram of the tunnel is shown in Figure 2.

A computer-controlled linear actuator was used to move the flat plate on the specified trajectories. The linear actuator was a LinTech ball screw with a diameter of 0.75 " and lead of 0.5", driven by a NEMA 34-490 microstepping motor. The position of the heaving plate was measured with an Encoder Products Company rotary encoder on the ball screw, with a resolution of 1000 lines per revolution. The flat plate was machined from aluminum to have semicircular leading and trailing edges, and mounted to be parallel to the freestream. In these experiments, the plate had length $c=10.1 \mathrm{~cm}$, span $b=45 \mathrm{~cm}$, and thickness ratio $d=1 / 16$. The plate's initial distance from the wall was $h_{0}=1.3 \mathrm{~cm}$. Due to mechanical limitations, a constant $t_{\text {accel }}$ of 0.36 seconds was used.

Particle image velocimetry (PIV) was used to measure the two-dimensional velocity fields around the plate using a LaVision PIV system with two Photron Fastcam APS-RX cameras. The cameras were aligned with the horizontal mid-plane of the test section, yielding a combined field of view as indicated in Figure 2. With a resolution of 1024 x 1024 pixels, 2048 images were recorded with both cameras in each experiment. Neutrally buoyant 10 micron hollow glass spheres were used for PIV seeding. These were illuminated at the test section's mid-plane with a 25mJ DM20-527 Photonics YAG laser, which had been expanded into a plane with a cylindrical lens. The images were processed in LaVision DaVis 8. 


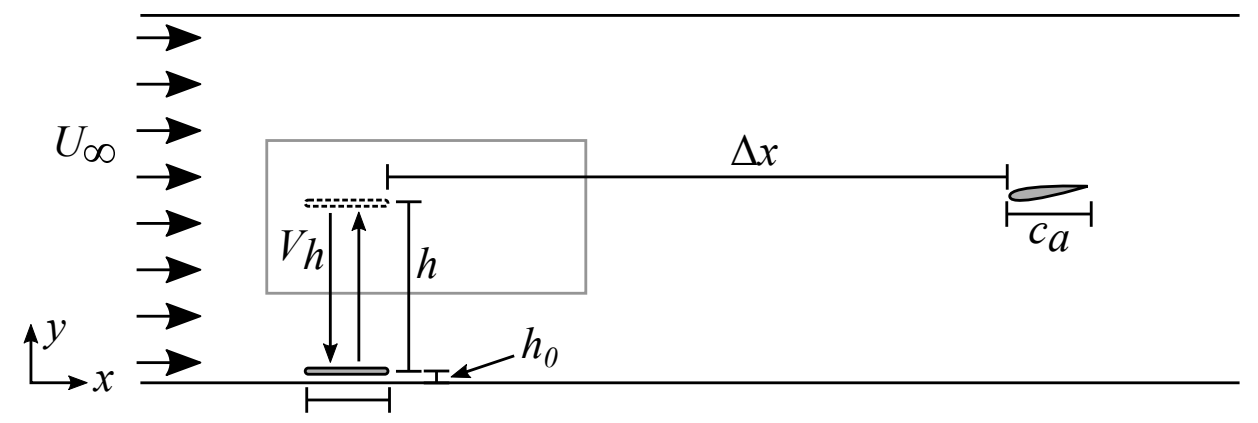

Figure 2: Diagram of the gusting system. The upper and lower lines are the sides of the water tunnel's test section. The gray rectangle indicates the PIV field of view. The axes shown are for definition of positive and negative directions.

A NACA 0018 airfoil was mounted to a force balance a distance of $\Delta x=75 \mathrm{~cm}$ downstream of the heaving plate at an angle of attack of $8^{\circ}$. The airfoil had a chord of $10 \mathrm{~cm}$ and wetted span of $45.7 \mathrm{~cm}$. A free surface plate was used to reduce the three-dimensionality of the flow around the airfoil. An adjustable portion allowed the airfoil to pass through the plate with a clearance of $3 \mathrm{~mm}$ on all sides. Force measurements on the airfoil employed an ATI Nano43 six-axis force transducer, sampled at $200 \mathrm{~Hz}$.

A custom Labview program synchronized the motion of the plate with measurement of its position, the forces on the airfoil, and the start of the PIV system.

\section{Experiments}

An initial parameter search was performed to cursorily examine the relative influences of the Reynolds number, $R e$, and heaving speed ratio, $S$. Experiments were performed for each combination of $R e=$ $(7500,13700,20000)$ and $S=(0.1,0.5,1.0)$, with heaving distance ratio $H=2.1$. For each of these, the PIV frequency was set to 130 time steps per $t_{c}$.

A second set of experiments investigated the relative influences of $S$ and the heaving time ratio, $T$, through variation of the heaving distance ratio, $H=T S$, at $R e=20,000$. Each combination of $S=(0.09,0.36,1.0)$ and $H=(1.6,1.9,2.1,2.4,2.6)$ was tested five times. The PIV system frequency was set to 125 time steps per $t_{c}$.

A third set of experiments measured the forces on the airfoil at a chordwise Reynolds number of 20,000 as it interacted with gusts generated over a range of $S$ and $H$. Each combination of $S=(0.1,0.25,0.5)$ and $H=(1.6,1.9,2.1,2.4,2.6)$ was tested five times for both positive and negative initial directions of plate motion. Forces were measured from $20 t_{c}$ before to $100 t_{c}$ after the estimated time of the gust reaching the airfoil.

\section{Results}

Examination of the first set of PIV fields showed little dependence on $R e$ over the tested range and strong variation with $S$. The slowest heaving resulted in attached flow over the plate while in motion, whereas the fastest had an extended separated region. This is consistent with the effective angle of attack of the plate, $\alpha_{\text {eff }}$, which took values of $6^{\circ}$ to $45^{\circ}$.

A typical example of the flow around the heaving plate is shown in Figure 3, where the black lines delineate vortical regions found with Graftieaux's $\Gamma_{2}$ function. ${ }^{22}$ In the rest of the paper, these regions will be referred to as vortices. Behind the upward moving plate (Figure 3b), a significant separated region was observed due to the high $\alpha_{e f f}=20^{\circ}$. Immediately after the plate changed directions (Figure $3 \mathrm{c}$ ), significant vorticity was released from the plate and began to roll up. After some time (Figure $3 \mathrm{~d}$ ) much of the positive vorticity moved downstream, having coalesced into a single vortex. After the plate came to rest (Figure 3e), additional vortical structures, formed due to deceleration and interaction with the wall, advected downstream. 
The circulation of the primary vortex, $\Gamma_{v}$, is displayed in Figure 4. For each set of experiments, $\Gamma_{v}$ was computed in two ways: as the average of $\Gamma_{v}$ from each of the five repetitions, or from the average of the five flow fields. These are plotted in Figure $4 \mathrm{a}$ as asterisks and circles, respectively. A linear fit yields $\Gamma_{v} \approx 0.85 \pi \mathrm{cUs}$, which differs from Equation 6 by the constant: 0.85 versus 1.0. Figure $4 \mathrm{~b}$ shows that $\Gamma_{v}$ does not vary consistently with $H$. No simple relationship between $\Gamma_{v}$ and $H$ or $T$ was found. Future experiments will expand the range of the parameters in order to search for such a relationship.

Examples of the forces resulting from the gust-airfoil interaction are shown in Figure 5. Figures 5a and 5c show the airfoil's lift coefficient due to interaction with a gust due to initial plate motion in the $+y$ direction, whereas Figures $5 \mathrm{~b}$ and $5 \mathrm{~d}$ are due to initial motion in the $-y$ direction. These motions generated vortices with positive and negative circulation, respectively. The central dashed lines indicate the estimated arrival time of the gust, which assumed that the gust departed the plate immediately after the direction change and traveled with the freestream. The regular oscillations in the upper plots were a result of von Karman vortex shedding, which appeared to be suppressed during the gust encounter. The lower plots were low-pass filtered at half of the shedding frequency to reveal the low-frequency behavior. The interaction with a vortex of positive circulation led to an initial sharp increase in the lift, which is opposite the effect of the vortex with negative circulation. The forces due to the vortices with opposite circulation are not symmetric, since the airfoil has a non-zero angle of attack. Across the repetitions of each type of gust-airfoil interaction, the similarity in the low-frequency behavior demonstrated that this apparatus created very repeatable gusts. The envelope of the shedding-related forces was also quite repeatable, though the shedding phase was not. Similarly to the experiments of Perrotta and Jones, ${ }^{13}$ the forces did not regain their unperturbed behavior until roughly $15-20 t_{c}$ after the gust interaction.

\section{Conclusions}

A simple vortical gust generation system has been built and tested. The circulation of these gusts was proportional to the heaving speed, as predicted by unsteady thin airfoil theory. This system demonstrated its repeatability through measurements of the gusts themselves as well as the forces on a downstream airfoil. In the future, further examination of the flow around the plate will improve understanding of the gust's structure and evolution. The ultimate goal is to use this gust generator to investigate the response of an airfoil to incoming vortical gusts.

\section{Acknowledgments}

This research is funded by the Gordon and Betty Moore Foundation through Grant GBMF\#2645 to the California Institute of Technology

\section{References}

\footnotetext{
${ }^{1}$ Eldredge, J. D., Wang, C., and Ol, M. V., "A computational study of a canonical pitch-up, pitch-down wing maneuver," AIAA Pap., Vol. 70, No. 8, 2009, pp. 3242-3250.

${ }^{2}$ Wagner, H., "Über die Entstehung des dynamischen Auftriebes von Tragflügeln," Zeitschrift für Angew. Math. und Mech., Vol. 5, No. 1, 1925, pp. 17-35. 1935.

${ }^{3}$ Theodorsen, T., "General theory of aerodynamic instability and the mechanism of flutter," NACA Annu. Rep., Vol. 20,

${ }^{4}$ Küssner, H. G., "Zusammenfassender Bericht über den instationären Auftrieb von Flügeln," Luftfahrtforschung, Vol. 13, No. 12, 1936, pp. 410-424.

${ }^{5}$ Garrick, I. E., "On some reciprocal relations in the theory of nonstationary flows," NACA Annu. Rep., Vol. $24,1938$.

${ }^{6}$ von Karman, T. and Sears, W. R., "Airfoil theory for non-uniform motion," J. Aeronaut. Sci., Vol. 5, No. $10,1938$.

${ }^{7}$ Administration, F. A., "Advisory circular 25.341-1: dynamic gust loads," Tech. rep., Federal Aviation Administration, 2014.

${ }^{8}$ Etele, J., "Overview of wind gust modelling with application to autonomous low-level UAV control," Tech. rep., 2006.

${ }^{9}$ Moulin, B. and Karpel, M., "Gust loads alleviation using special control surfaces," J. Aircr., Vol. 44, No. 1, 2007, pp. $17-25$.

${ }^{10}$ Kerstens, W., Williams, D. R., Pfeiffer, J., King, R., and Colonius, T., "Closed loop control of a wing's lift for 'gust' suppression," 5th Flow Control Conf., 2010, pp. 1-11.

${ }^{11}$ Brion, V., Lepage, A., Amosse, Y., Soulevant, D., Senecat, P., Abart, J. C., and Paillart, P., "Generation of vertical gusts in a transonic wind tunnel," Exp. Fluids, Vol. 56, No. 145, 2015.
} 


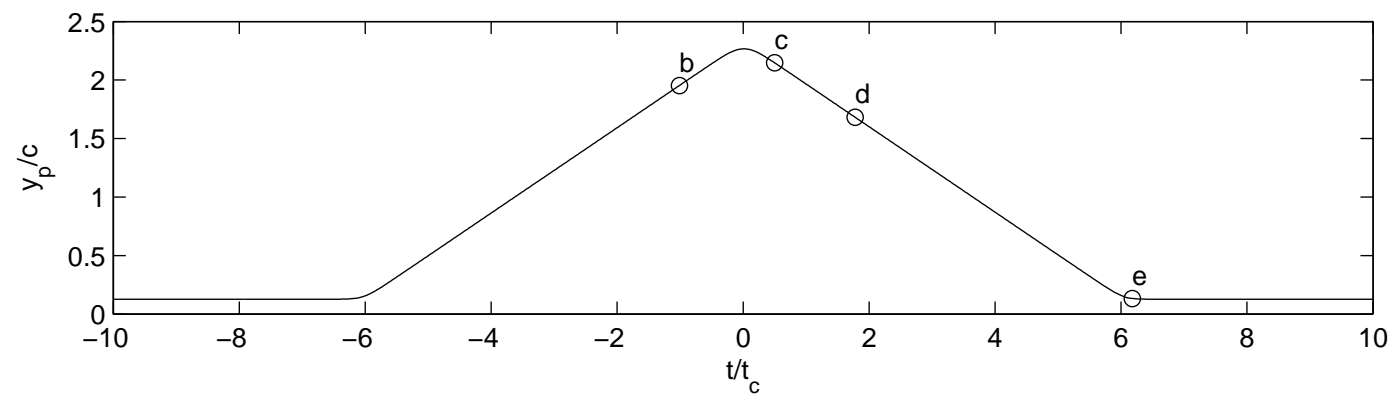

(a) The plate position over time. The times of the following vorticity fields are labeled on this plot.

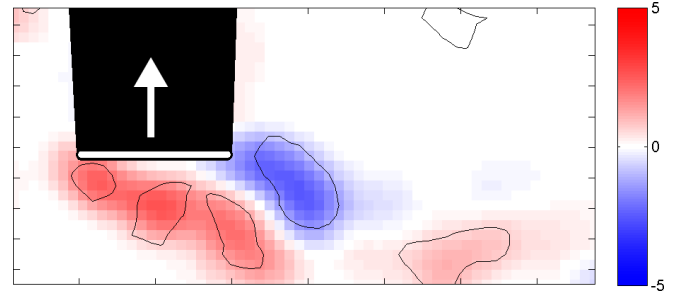

(b) The plate is moving steadily upward, with a wake trailing behind.

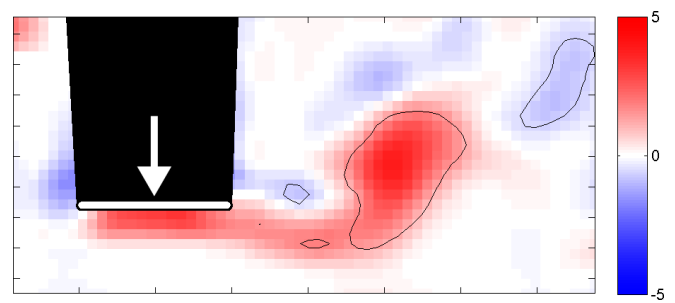

(d) The plate moves steadily downward, and the vortex continues to roll up.

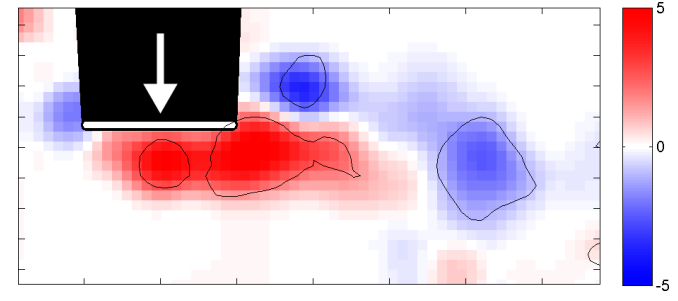

(c) The plate has changed directions, and the wake is rolling up.

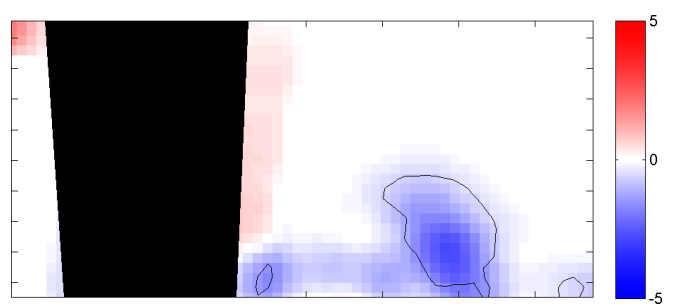

(e) The plate has stopped moving, and has shed vorticity due to its deceleration and possible wall interactions.

Figure 3: Subplot $a$ shows the position of the plate over time for experiments with $S=0.36$ and $T=0.17$. Subplots $b-e$ show the vorticity field around the moving plate, averaged over five repetitions. The black contours delineate where the $\Gamma_{2}$ function identified vortices. The solid black region is the area in the shadow of the plate where PIV data is unavailable. The white arrow denotes the direction of the plate's motion. 


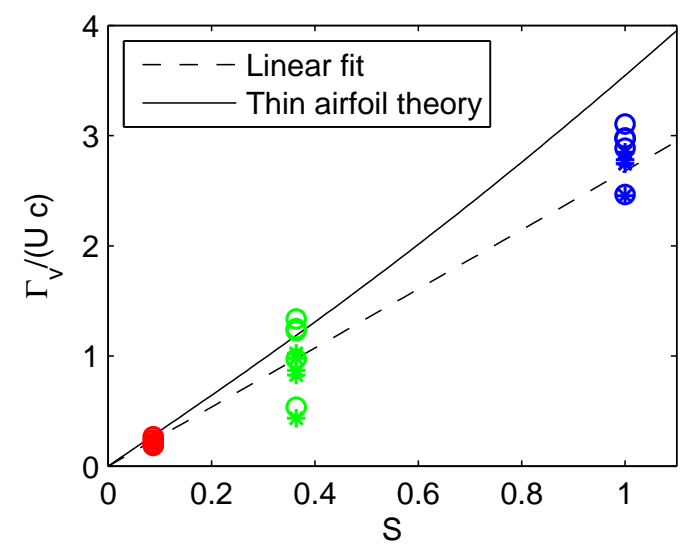

(a) Vortex strength as a function of $S$, the dimensionless heaving speed.

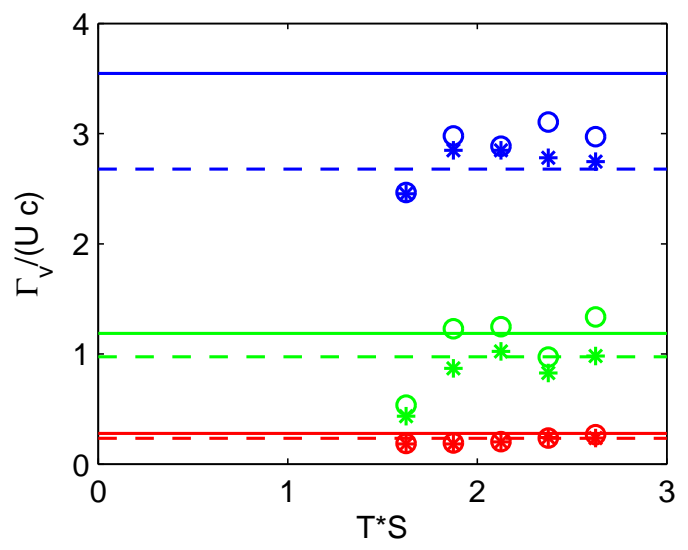

(b) Vortex strength as a function of $T S=H$, the dimensionless heaving distance.

Figure 4: Solid lines indicate the estimated vortex strength from unsteady thin airfoil theory. Dashed lines are the linear fit with with $S$. Symbols and lines are colored with respect to $S$, where red, green, and blue are $S=0.09,0.36$, and 1.0, respectively. Asterisks indicate the mean of $\Gamma_{v}$ computed across five repetitions of the experiment. Circles represent $\Gamma_{v}$ computed from the average PIV field of these experiments.

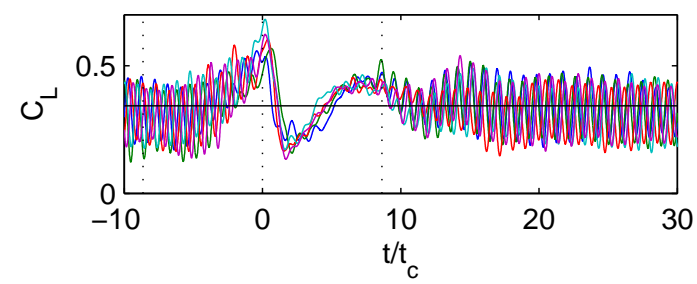

(a) $C_{L}$ with a vortex of positive circulation.

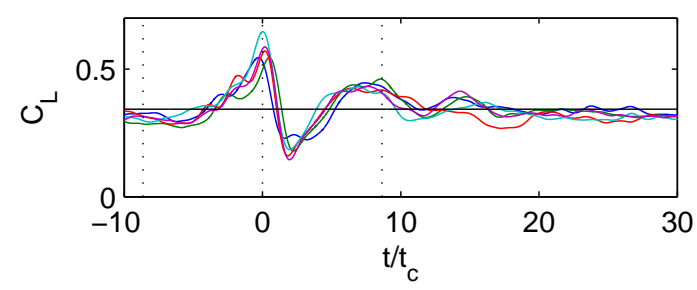

(c) $C_{L}$ with a vortex of positive circulation, low-pass filtered.

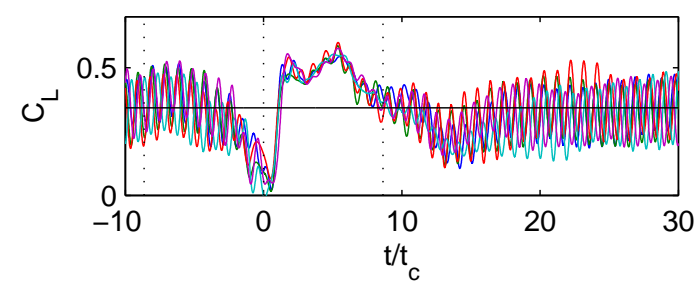

(b) $C_{L}$ with a vortex of negative circulation.

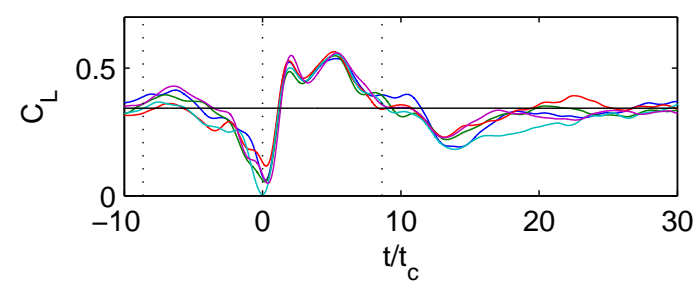

(d) $C_{L}$ with a vortex of negative circulation, low-pass filtered.

Figure 5: $C_{L}$ traces for the interaction of generated gusts with the airfoil at $8^{\circ}$. The gust generator had $S=0.25$ and $T=8.5$. The solid line is the average unperturbed $C_{L}$. The dashed lines indicate the times of the start, peak, and end of the plate motion, shifted by the gust's estimated travel time. The different colors indicate the five repetitions for each experiment. Subplots $a$ and $c$ had upward initial plate motion, whereas $b$ and $d$ had downward initial motion. 
${ }^{12}$ Buell, D. A., "An experimental investigation of the velocity fluctuations behind oscillating vanes," Tech. rep., National Aeronautics and Space Administration, 1969.

${ }^{13}$ Perrotta, G. and Jones, A. R., "Transient aerodynamics of large transverse gusts and geometrically similar maneuvers," 54th AIAA Aerosp. Sci. Meet., 2016, p. 2074.

${ }^{14}$ Lua, K. B., Lim, T. T., Yeo, K. S., and Oo, G. Y., "Wake-structure formation of a heaving two-dimensional elliptic airfoil," AIAA J., Vol. 45, No. 7, 2007, pp. 1571-1583.

${ }^{15}$ Widmann, A. and Tropea, C., "Parameters influencing vortex growth and detachment on unsteady aerodynamic profiles," J. Fluid Mech., Vol. 773, 2015, pp. 432-459.

${ }^{16}$ Chen, J. M. and Fang, Y.-C., "Strouhal numbers of inclined flat plates," J. Wind Eng. Ind. Aerodyn., Vol. 61, 1996, pp. $99-112$

${ }^{17}$ Akkala, J. M., Eslam Panah, A., and Buchholz, J. H., "Vortex dynamics and performance of flexible and rigid plunging airfoils," J. Fluids Struct., Vol. 54, 2015, pp. 103-121.

${ }^{18}$ Munk, M. M., "General Theory of Thin Wing Sections," NACA Annu. Rep., Vol. 8, 1922.

${ }^{19}$ Kutta, W. M., "Auftriebskräfte in strömenden Flüssigkeiten," Illus. Aeronaut. Mitteilungen, Vol. 6, No. 133, 1902, pp. $133-135$

${ }^{20}$ Joukowski, N., "On annexed vortices," Trans. Phys. Sect. Imp. Soc. Friends Nat. Sci. Moscow, Vol. 13, No. 2, 1906, pp. $12-25$.

${ }^{21}$ Thomson, W., "On vortex motion," Trans. R. Soc. Edinburgh, Vol. 25, No. 01, 1868, pp. 217-260.

${ }^{22}$ Graftieaux, L., Michard, M., and Grosjean, N., "Combining PIV, POD and vortex identification algorithms for the study of unsteady turbulent swirling flows," Meas. Sci. Technol., Vol. 12, No. 9, 2001, pp. 1422-1429. 